\title{
Reliability Analysis of Communicating Recovery Blocks
}

\author{
Oded Berman \\ University of Toronto, Toronto \\ U. Dinesh Kumar \\ University of Exeter, Exeter
}

Key Words - Communicating recovery block, Domino effect, Fault tolerance, Optimization, Software reliability.

Summary \& Conclusions - This paper studies 2 models for communicating recovery blocks (RB) with statisticallyindependently failing software versions where versions are executed sequentially. Model \#1 considers 2 RB: RB-1 \& RB-2, where RB-2 receives some data from RB-1. Thus, if a version in RB-2 fails then RB-1 has to rollback to its initial state. Model \#2 considers 2 RB in conversation: both blocks must satisfy their respective acceptance tests before any of the blocks are allowed to exit from the conversation. Simple expressions for the reliability of the system are derived for models \#1 \& \#2, and it is proved for them that the reliability of a RB consisting of versions ordered from smallest to largest based on failure probability is as reliable as any other list of the versions. Optimization models are developed for models \#1 \& \#2. The paper presents efficient branch \& bound procedures to solve the optimization models.

\section{INTRODUCTION}

$\begin{array}{cl}\text { Acronyms }^{1} & \\ \text { B\&B } & \text { branch and bound } \\ \text { RB } & \text { recovery block } \\ \text { CRB } & \text { communicating RB } \\ \text { DRB } & \text { distributed RB } \\ \text { LB, UB } & \text { [lower, upper] bound } \\ \text { TestSeg } & \text { testing; segment }\end{array}$

One approach to overcoming software failures is to use $\mathrm{RB}[3,6,8-10,12,14]$. In a $\mathrm{RB}$, there exist several versions of a program and an acceptance test. Upon invocation of a $\mathrm{RB}$, a primary version is executed first and the output is then submitted to an acceptance test. Whenever an output is rejected by the acceptance test, the succeeding version is executed. This procedure is continued until: 1) an output of a version is accepted, or 2) the output of the last version is rejected.

If the software system consists of many $\mathrm{RB}$ which do not interact with each other, then the recovery of the input state upon failure of a version in a RB is purely local to the failing RB. However, if two or more RB interact in some way, then the state recovery becomes complicated by the

\footnotetext{
IThe singular \& plural of an acronym are always spelled the same.
}

fact that the state recovery in one RB might be propagated to another RB. For example, consider two RB, RB-1 \& RB2. Let RB-1 generate some data and transmit it to RB-2. If an error occurs in RB-2 (and hence RB-2 is restored to its initial state) then it might be necessary to restore RB-1 also to its initial state, even though the TestSeg accepts the output from RB-1. Suppose that there are several such CRB. Then the state recovery might be propagated to all the RB upon failure of a single RB. In some cases an uncontrollable activity might propagate through the system in a kind of domino effect $[6,12,13]$. One method of avoiding a domino effect and coordinating state recovery of CRB is by enclosing interactions in conversation $[12,13]$.

An initial study on CRB (also called DRB) was done by Randell [12], which presents techniques for structuring CRB and RB in conversation. Russel [13] developed sufficient conditions for a system to be domino free, and obtained bounds on the amount of unnecessary state restoration for certain classes of systems. Kim [7] provides a detailed study on DRB as an approach for realizing both hardware \& software fault tolerance in realtime distributed and parallel computer systems. Ref [7] also discusses the application and limitations of the DRB scheme for local area network (LAN) based systems and highly parallel multi-computer network (HPM) based systems. Dugan [4] presents two models, one based on fault tree and the other on Markov process for analyzing DRB; her paper presents simple expressions for failure of $D R B$ by considering:

- failure of both primary \& secondary versions because of two unrelated or a single related fault;

- failure of hardware;

- TestSeg fails to detect unacceptable result or to accept correct result.

Although [4] gives a good insight about the CRB, the analysis is restricted to two versions, and the expressions can not be used directly for optimization. Both fault tree and Markov models for reliability prediction become computationally complex as the number of versions increases.

Optimization models for RB were developed by Belli \& Jedrzejowicz [3] and later in [1]. Models for optimal checkpointing frequency that minimizes the mean execution time were developed by Nicola [11]. Ref [1] presents two optimization models for an independent $\mathrm{RB}$ and a 
consensus RB.

This paper extends our earlier work [1] to CRB. We analyze two models of CRB. Model 1 considers two interacting RB, RB-1 \& RB-2, with domino effect; model 2 deals with two RB in conversation (domino free). For both models, the paper:

- derives explicit expressions for reliability

- proves that the reliability. when the versions are ordered from the smallest to the largest based on failure probabilities in each RB, is at least as reliable as any other order of the versions.

- presents optimization models for both models.

Efficient B\&B procedures are developed to solve the optimization problems.

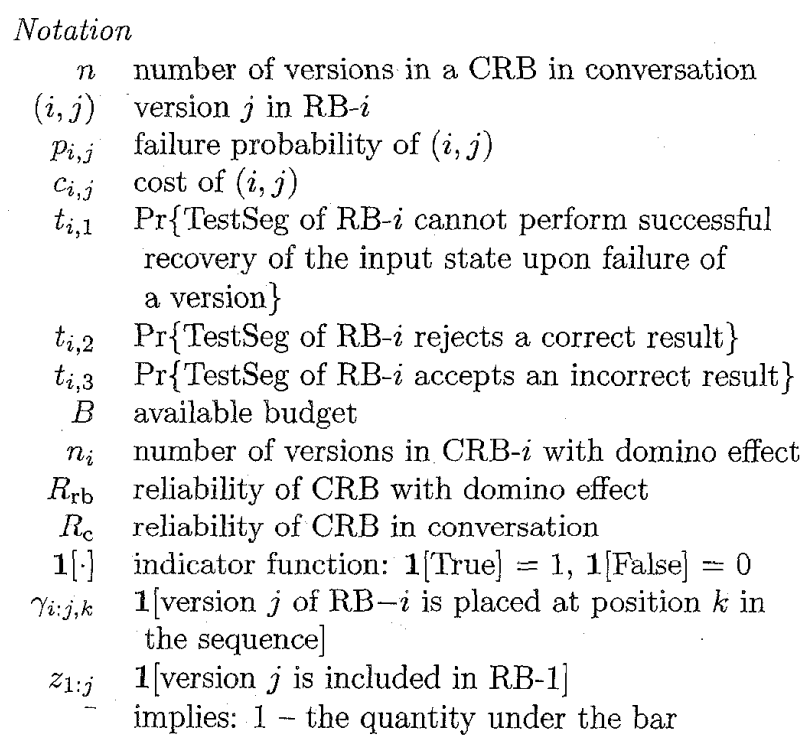

\section{General Assumptions}

1. Different versions in a RB are mutually s-independent.

2. Versions in a RB are executed sequentially starting from the primary version (or first version).

3. All versions produce an output: the software does not stop executing without producing an output.

4. Failure of common platform services such as communication network, operating system, and device drivers are ignored.

\section{CRB WITH DOMINO EFFECT}

This section considers the problem with the following additional assumptions; $\mathrm{A} 2$ - A5 simplify the presentation.

A1. CRB has the domino effect: state restoration in one block can force all the other interacting $R B$ to return to their initial state.

A2. There are 2 RB (RB-1 \& RB-2).

A3. $\mathrm{RB}-i$ has $n_{i} s$-independent versions, $i=1,2$; where $n_{1}=n_{2}=n$.

A4. RB-1 generates and transmits data to RB-2. Upon invocation, RB-1 \& RB-2 run concurrently.
A5. In case of error in the primary version of RB-2, the TestSeg activates the first alternative version. This forces RB-1 also to be restored: RB-1 is also run using an alternative version, even though the output from the primary version of RB-1 is accepted by the TestSeg.

A6. RB-1 transfers data to RB-2 only after the acceptance test accepts the output of a version in $\mathrm{RB}-1$.

\section{Notation for Section 2}

$x_{i, j}$ event: version $j$ of $\mathrm{RB}-i$ produces:

1) an incorrect result, and the TestSeg rejects it, or 2) a correct result and the TestSeg rejects it; in either case, the TestSeg performs a successful recovery

$y_{i, j} \quad$ event: version $j$ of RB- $i$ produces correct output and the TestSeg accepts the correct output

$R_{1}^{i+} \quad$ reliability of RB-1 (in absence of RB-2) with versions $\{i, i+1, \ldots, n\}, i \geq 2$

$R_{1}^{i-} \quad$ reliability of $R B-1$ (in absence of RB-2) with versions $\{1,2, \ldots, i-1\}$ when all versions except $i-1$ fail, $i \geq 2$

$R_{1}^{i, k} \quad$ reliability of $\mathrm{RB}-1$ (in absence of $\mathrm{RB}-2$ ) with versions $\{i, i+1, \ldots, k-1\}$ when all versions except $k-1$ fail, $i \geq 2$

Following [1], the corresponding probabilities for this model are:

$$
\begin{aligned}
& \operatorname{Pr}\left\{y_{i, j}\right\}=\bar{p}_{i, j} \cdot \bar{t}_{i, 2} \\
& \operatorname{Pr}\left\{x_{i, j}\right\}=\bar{t}_{i, 1} \cdot\left[p_{i, j} \cdot \bar{t}_{i, 3}+\bar{p}_{i, j} \cdot t_{i, 2}\right]
\end{aligned}
$$

The reliability of RB-1 in the absence of $R B-2$ is [1]:

$$
R_{1}=\operatorname{Pr}\left\{y_{1,1}\right\}+\sum_{j=1}^{n-1}\left[\prod_{k=1}^{j} \operatorname{Pr}\left\{x_{1, k}\right\}\right] \cdot \operatorname{Pr}\left\{y_{1, j+1}\right\}
$$

$$
\begin{aligned}
R_{\mathrm{rb}} & =\operatorname{Pr}\left\{y_{1,1}\right\} \cdot \operatorname{Pr}\left\{y_{2,1}\right\}, \text { for } n=1 \\
R_{\mathrm{rb}} & =\operatorname{Pr}\left\{y_{1,1}\right\} \cdot \operatorname{Pr}\left\{y_{2,1}\right\}+\operatorname{Pr}\left\{x_{1,1}\right\} \cdot \operatorname{Pr}\left\{y_{1,2}\right\} \cdot \operatorname{Pr}\left\{y_{2,1}\right\} \\
& +\operatorname{Pr}\left\{y_{1,1}\right\} \cdot \operatorname{Pr}\left\{x_{2,1}\right\} \cdot \operatorname{Pr}\left\{y_{1,2}\right\} \cdot \operatorname{Pr}\left\{y_{2,2}\right\}, \text { for } n=2
\end{aligned}
$$

In general, the reliability of a CRB can be obtained as follows:

$$
\begin{aligned}
& R_{1}^{i+} \equiv \operatorname{Pr}\left\{y_{1, i}\right\}+\sum_{j=i}^{n-1}\left[\prod_{k=i}^{j} \operatorname{Pr}\left\{x_{1, k}\right\}\right] \cdot \operatorname{Pr}\left\{y_{1, j+1}\right\} \\
& R_{1}^{i-} \equiv\left[\prod_{k=1}^{i-2} \operatorname{Pr}\left\{x_{1, k}\right\}\right] \cdot \operatorname{Pr}\left\{y_{1, i-1}\right\} \\
& R_{1}^{i, k} \equiv\left[\prod_{j=i}^{k-2} \operatorname{Pr}\left\{x_{1, j}\right\}\right] \cdot \operatorname{Pr}\left\{y_{1, k-1}\right\}, \text { for } k \neq i
\end{aligned}
$$

$R_{1}^{i, i} \equiv 0$ 


$$
\begin{aligned}
& R_{\mathrm{rb}}=R_{1} \cdot \operatorname{Pr}\left\{y_{2,1}\right\}+\sum_{k=1}^{n-1} \sum_{j_{1}=2}^{n-k+1} \sum_{j_{2}=j_{1}+1}^{n-k} \ldots \sum_{j_{k}=j_{k-1}+1}^{n} \\
& R_{1}^{j_{1}^{-}} \cdot R_{1}^{j_{1}, j_{2}} \cdots R_{1}^{j_{k-1}, j_{k}} \cdot R_{1}^{j_{k}^{+}} \cdot\left[\prod_{j=1}^{k} \operatorname{Pr}\left\{x_{2, j}\right\}\right] \\
& \quad \operatorname{Pr}\left\{y_{2, k+1}\right\}
\end{aligned}
$$

$\mathrm{Eq}(5)$ reflects that:

- upon invocation of CRB, both RB-1 \& RB-2 are executed sequentially starting from version 1 to version $n$, - for successful completion of the program, an output of a version in RB-1 must be accepted followed by the acceptance of an output of a version in RB-2.

The number of outputs accepted in RB-1 is equal to the number of versions executed in RB-2.

Theorem 1. For CRB with $n s$-independent versions in each block, the list ordered from smallest to largest based on failure probabilities is at least as reliable as any other list of the versions.

\subsection{Mathematical Programming Formulation}

$$
\begin{aligned}
& \text { Redefine } R_{1}, R_{1}^{i-}, R_{1}^{i+}, R_{1}^{i, k} \text {. } \\
& R_{1} \equiv \sum_{j=0}^{n-1}\left[\prod_{k=0}^{j} \operatorname{Pr}\left\{x_{1, k}\right\}^{z_{1: k}}\right] \cdot \operatorname{Pr}\left\{y_{1, j+1}\right\}^{z_{1: j+1}} \\
& -\sum_{j=0}^{n-1} \bar{z}_{1, j+1} \cdot\left[\prod_{k=0}^{j} \operatorname{Pr}\left\{x_{1, k}\right\}^{z_{1: k}}\right] \cdot \operatorname{Pr}\left\{y_{1, j+1}\right\}^{z_{1: j+1}} \\
& \operatorname{Pr}\left\{x_{1,0}\right\} \equiv 0, \quad z_{1: 0} \equiv 1 ; \\
& R_{1}^{i-} \equiv\left[\prod_{k=1}^{i-2} \operatorname{Pr}\left\{x_{1, k}\right\}^{z_{1: k}}\right] \cdot \operatorname{Pr}\left\{y_{1, i-1}\right\}^{z_{1: i-1}} \\
& -\bar{z}_{1, i-1} \cdot\left[\prod_{k=1}^{i-2} \operatorname{Pr}\left\{x_{1, k}\right\}^{z_{1: k}}\right] \cdot \operatorname{Pr}\left\{y_{1, i-1}\right\}^{z_{1: i-1}} \\
& R_{1}^{i, k} \equiv\left[\prod_{j=i}^{k-2} \operatorname{Pr}\left\{x_{1, j}\right\}^{z_{1: j}}\right] \cdot \operatorname{Pr}\left\{y_{1, k-1}\right\}^{z_{1: k-1}} \\
& -\bar{z}_{1, k-1} \cdot\left[\prod_{j=i}^{k-2} \operatorname{Pr}\left\{x_{1, j}\right\}^{z_{1: j}}\right] \cdot \operatorname{Pr}\left\{y_{1, k-1}\right\}^{z_{1: k-1}} \\
& R_{1}^{i+} \equiv \sum_{j=i}^{n}\left[\prod_{k=1}^{j-1} \operatorname{Pr}\left\{x_{1, k}\right\}^{z_{1: k}}\right] \cdot \operatorname{Pr}\left\{y_{1, j}\right\}^{z_{1: j}} \\
& -\bar{z}_{1, j} \cdot\left[\prod_{k=1}^{j-1} \operatorname{Pr}\left\{x_{1, k}\right\}^{z_{1: k}}\right] \cdot \operatorname{Pr}\left\{y_{1, j}\right\}^{z_{1: j}}
\end{aligned}
$$

The reliability of inter-dependent $\mathrm{RB}$ is:

$$
\begin{aligned}
R_{\mathrm{rb}} & =\sum_{k=1}^{m-1} \sum_{j_{1}=2}^{n-k+1} \sum_{j_{2}=j_{1}+1}^{n-k} \ldots \sum_{j_{k}=j_{k-1+1}}^{n} R_{1}^{j_{1}^{-}} \\
& \cdot R_{1}^{j_{1}, j_{2}} \cdots R_{1}^{j_{k-1}, j_{k}} \cdot R_{1}^{j_{k}^{+}} \cdot U_{k} \cdot\left[\sum_{i=1}^{n} \operatorname{Pr}\left\{y_{2, i}\right\} \cdot \gamma_{2: i, k+1}\right] \\
& +R_{1} \cdot \sum_{i=1}^{n} \operatorname{Pr}\left\{y_{2, i}\right\} \cdot \gamma_{2: i, 1} \\
U_{k} & \equiv \prod_{j=1}^{k}\left[\sum_{i=1}^{n} \operatorname{Pr}\left\{x_{2, i}\right\} \cdot \gamma_{2: i, j}\right], \\
m & \equiv \sum_{i=1}^{n} \sum_{k=1}^{n} \gamma_{2: i, k}
\end{aligned}
$$

The corresponding optimization problem is:

$\max \left[R_{\mathrm{rb}}\right]$

Subject to:

$\sum_{i=1}^{n} c_{1, i} \cdot z_{1: i}+\sum_{j=1}^{n} \sum_{k=1}^{n} c_{2, j} \cdot \gamma_{2: j, k} \leq B$

$\sum_{i=1}^{n} \gamma_{2: i, k} \leq 1$, for $k=1,2, \ldots, n$

$\sum_{k=1}^{n} \gamma_{2: i, k} \leq 1$, for $i=1,2, \ldots, n$

- The objective function (11) maximizes reliability.

- Constraint (12) guarantees that the total expenditure is no more than the available budget.

. Constraint (13) reflects that if a version is executed in RB-2 it occupies at most 1 position.

- Constraint (14) reflects that each position can be occupied by at most 1 version in the block \#2.

\subsection{The B\&B Procedure}

The optimization problem in section 2.1 can be solved using an efficient B\&B technique. Without any loss of generality, in each block let the versions be numbered based on their rankings, beginning with the most reliable version (smallest probability of failure).

Step 1 Choose Versions of Block \#1

- At tree-level \#1, it is determined whether version \#1 of block \#1 should be selected $\left(z_{1: 1}=1\right)$ or not $\left(z_{1: 1}=0\right)$.

- At tree-level \#2, it is determined whether version \#2 of block \#1 should be selected $\left(z_{1: 2}=1\right)$ or not $\left(z_{1: 2}=0\right)$.

- And so on.

When all versions of block \#1 have been considered, then consider block \#2.

Step 2 Choose Versions of Block \#2

1. At tree-level \#n+1, version \#1 is selected

$\left(\gamma_{2: 1,1}=1, \gamma_{2: 1, k}=0\right.$ for $\left.k=2, \ldots, n\right)$, 
or not selected

$\left(\gamma_{2: 1, k}=0\right.$ for $\left.k=1, \ldots, n\right)$.

2. At tree-level \#2:

If version \#1 was selected then version \#2 is selected

$\left(\gamma_{2: 2,1}=0, \gamma_{2: 2,2}=1\right.$, and $\gamma_{2: 2, k}=0$ for $\left.k=3, \ldots, n\right)$, or not selected

$\left(\gamma_{2: 2, k}=0\right.$, for $\left.k=1,2, \ldots, n\right)$.

If version \#1 was not selected then version \#2 is selected

$\left(\gamma_{2: 2,1}=1, \gamma_{2: 2, k}=0\right.$ for $\left.k=2, \ldots, n\right)$,

or not selected

$\left(\gamma_{2: 2, k}=0\right.$ for $\mathrm{k}=1,2, \mathrm{n}$.).

3. At tree-levels \#3, ...:

Continue until all versions of block \#2 are considered.

For each partial solution, LB \& UB are computed. A partial solution can be eliminated (fathomed) from further consideration if:

i. its UB is less than or equal to the best LB available,

ii. the partial solution is unfeasible,

iii. the partial solution's $L B=U B$, in which case the partial solution is feasible and its value can be compared to the best LB available.

Theorem 1 is used in our structure of the $B \& B$ tree.

The LB \& UB for a node representing a partial solution are obtained in sections 2.2.1 \& 2.2.2.

\section{Notation}

$L_{i} \quad$ the set of versions in $\mathrm{RB}-i, i=1,2$

$P_{h} \quad h=1,2$ : any partial solution

$P_{1} \quad\left\{z_{1: 1}, z_{1: 2}, \ldots, z_{1: j}\right\}$

$P_{2}\left\{z_{1: 1}, z_{1: 2}, \ldots, z_{1: n}, \gamma_{2: 1,1}, \ldots \gamma_{2: 1, n}, \ldots\right.$,

$$
\left.\ldots \gamma_{2: i, 1}, \ldots \gamma_{2: i, n}\right\}
$$

\subsubsection{Upper bound}

The UB is the reliability of the best possible solution, including the partial solution that ignores cost. This is given by:

$\left\{z_{1: 1}, z_{1: 2}, \ldots, z_{1: j} ; z_{1: k}=1\right.$ for $k=j+1, \ldots, n ; \gamma_{2: j, j}=1$ for $j=1, \ldots, n\}$ for $P_{1}$;

$\left\{z_{1: j}\right.$ for $j=1, \ldots, n ; \gamma_{2: j, k}$ for $k=1, \ldots, i$ and $k=$ $1, \ldots, n ; \gamma_{2: i+j, m_{1}+j}=1$ for $\left.j=1, \ldots, n-i\right\}$ for $P_{2}$.

$m_{1} \equiv \sum_{j=1}^{i} \sum_{k=1}^{n} \gamma_{2: j, k}$.

\subsubsection{Lower bound}

The LB for $P_{1}$ or $P_{2}$ is the objective function value of the cheapest feasible solution that includes the partial solution. For $P_{2}$ it can be derived as follows.

1. Compute $T=$ set of versions in $L_{1} \cup L_{2}$ other than the versions in the partial solution.

2. Compute $T^{\prime}=\{[1],[2], \ldots,[n-i]\}=$ list of versions $T$ that include at least 1 version from each block, ordered from smallest cost to largest cost.
3. Derive $[r] \in T^{\prime}$, such that,

$$
\begin{aligned}
& \Psi_{n}+\sum_{k=[1]}^{[r]} c_{2, k} \leq B \\
& \Psi_{n}+\left[\sum_{k=[1]}^{[r+1]} c_{2, k}\right]>B \\
& \Psi_{n} \equiv \sum_{i=1}^{n}\left[z_{1: j} \cdot c_{1, j}+\sum_{j=1}^{i} \sum_{k=1}^{n} \cdot c_{2, j} \cdot \gamma_{2: j, k}\right]
\end{aligned}
$$

The LB is the reliability of versions:

$\left\{z_{1: j}\right.$ for $j=1, \ldots, n$;

$\gamma_{2: k, j}$ for $k=1, \ldots, i$ and $\left.j=1, \ldots, n\right\}$;

$\gamma_{2:[j], m_{1}+j}=1$ for $\left.j=1, \ldots, r\right\}$.

2.3 Example

Consider RB-1 \& RB-2, with $n=4$ versions, and $\mathrm{B}=46$. Other parameters are:

\begin{tabular}{ccccc}
$i$ & 1 & 2 & 3 & 4 \\
\hline$p_{1, i}$ & 0.1 & 0.15 & 0.25 & 0.30 \\
$c_{1, i}$ & 9 & 8 & 6 & 3 \\
$p_{2, i}$ & 0.05 & 0.15 & 0.20 & 0.25 \\
$c_{2, i}$ & 11 & 9 & 7 & 4 \\
\hline$t_{i, 1}=0.01, t_{i, 2}=0.05, t_{i, 3}=0.01$
\end{tabular}

When all the versions are included, then

\begin{tabular}{lllll}
$i$ & 1 & 2 & 3 & 4 \\
\hline $\operatorname{Pr}\left\{y_{1, i}\right\}$ & .8850 & .8075 & .7125 & .6650 \\
$\operatorname{Pr}\left\{x_{1, i}\right\}$ & .1425 & .1890 & .2821 & .3286 \\
$\operatorname{Pr}\left\{y_{2, i}\right\}$ & .9025 & .8075 & .7600 & .7125 \\
$\operatorname{Pr}\left\{x_{2, i}\right\}$ & .0960 & .1890 & .2356 & .2821
\end{tabular}

This problem can be solved using the $B \& B$ method in section 2.2, and is depicted in figure 1 .

For example, consider the partial solution, $z_{1: 1}$. The UB for $z_{1: 1}$ is the value of the (unfeasible) solution:

$\left\{z_{1: j}\right\}=1, \gamma_{2: j, j}=1$ for $\left.j=1,2,3,4\right\}$

and, from (10) the UB $=0.982661$.

The LB for this partial solution is derived as follows: Identify the cheapest feasible solution (total cost not to exceed 46) that includes $\left\{z_{1: 1}\right\}$ and at least one version from RB-2. This solution is:

$$
\begin{aligned}
& \left\{z_{1: j}\right\}=1, \text { for } j=1,2,3,4 \\
& \left.\quad \text { and } \gamma_{2: 2,1}=\gamma_{2: 3,2}=\gamma_{2: 4,3}=1\right\}
\end{aligned}
$$

and, from $(10)$ the $L B=0.963712$.

Similarly, one can derive LB \& UB for all the other partial solutions. In figure 1 , the partial solutions marked $*$, **, ***, correspond to fathoming of a partial solution due to the three reasons $1,2,3$ discussed in section 2.2 . The first time that 'an improved best LB is identified' is at node 11 corresponding to the feasible solution: $\left\{z_{1: j}\right\}=1$ for $j=1,2,3,4$; and $\left.\gamma_{2: 1,1}=\gamma_{2: 2,2}=1\right\}$ with $L B=0.97122$ (which is also the optimal solution). 


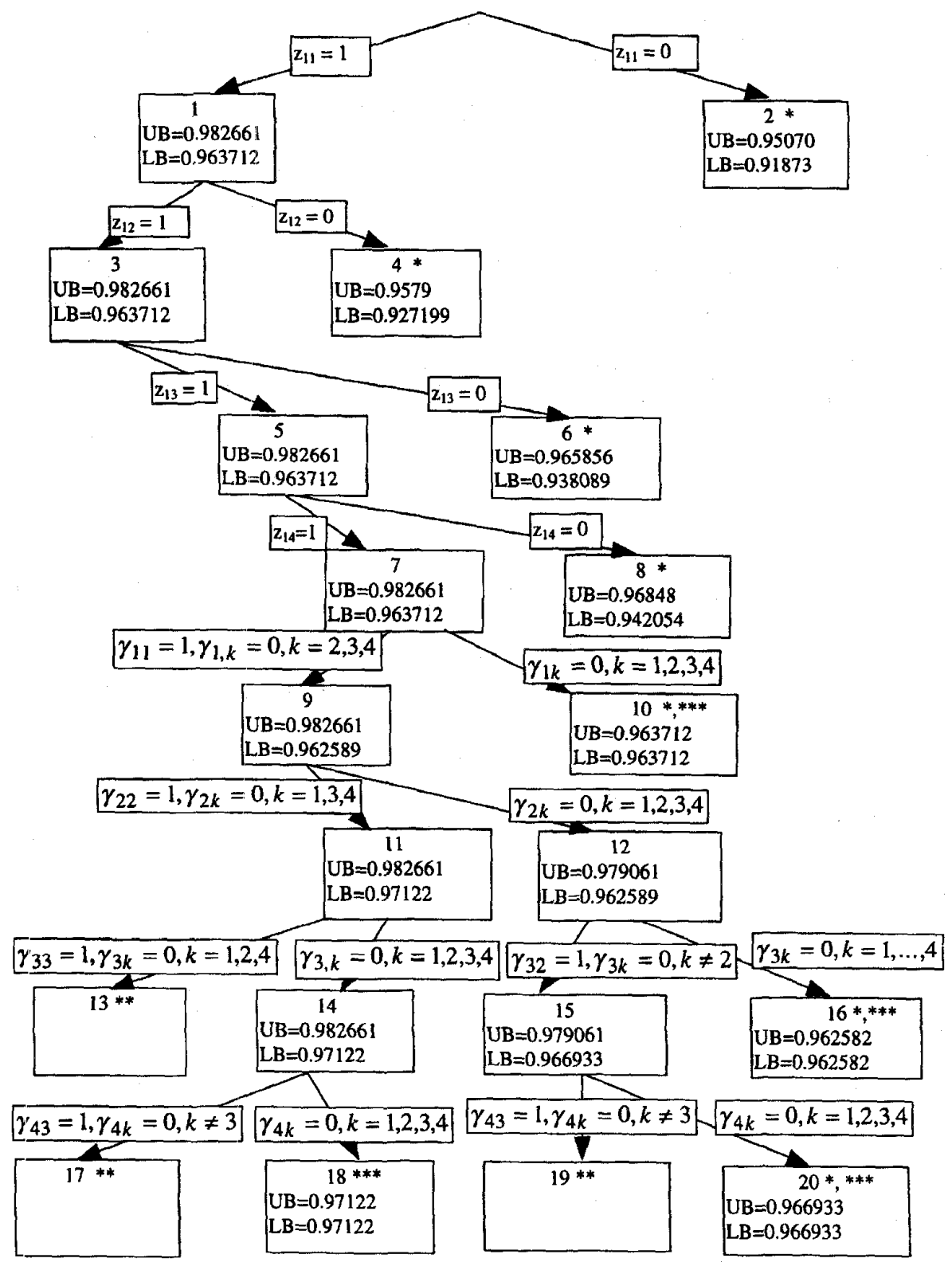

Figure 1: The B\&B for Example 2.3 Which Has CRB and the Domino Effect

[The first subscript, 2 , in $\gamma_{2: j, k}$ is omitted in the figure.]

This is the reason. why a $*$ is inserted also under nodes $6,10,20$.

\section{CRB IN CONVERSATION}

CRB with domino effect can result in an avalanche of state restoration, which can even lead to the initial state of the system. If: the acceptance tests of RB are established independently and if there is no restriction imposed on the message exchanged between the $\mathrm{RB}$, then a domino effect is always possible. One approach to coordinate state recovery (and hence avoid a domino effect) in an interdependent $\mathrm{RB}$ is by enclosing process interactions in a conversation. In a conversation, all blocks engaged in that conversation must satisfy their respective acceptance tests before any of the processes are allowed to exit the conversation. If the acceptance test of any $R B$ fails, all the $R B$ 
in the conversation must return to the point when they entered the conversation in order to resume execution.

Consider 2 RB (RB-1 and RB-2) in conversation. Assume that each block has $n s$-independent versions. Let $U^{i}$ be $\operatorname{Pr}\{$ output from at least 1 of version $i$ in RB- 1 or RB-2 is rejected by the acceptance test $\}$ :

$$
\begin{aligned}
U^{i} & \equiv \operatorname{Pr}\left\{x_{1, i}\right\} \cdot \operatorname{Pr}\left\{x_{2, i}\right\}+\operatorname{Pr}\left\{y_{1, i}\right\} \cdot \operatorname{Pr}\left\{x_{2, i}\right\} \\
& +\operatorname{Pr}\left\{x_{1, i}\right\} \cdot \operatorname{Pr}\left\{y_{2, i}\right\}
\end{aligned}
$$

The reliability of two RB in conversation is:

$$
\begin{aligned}
R_{\mathrm{c}} & =\operatorname{Pr}\left\{y_{1,1}\right\} \cdot \operatorname{Pr}\left\{y_{2,1}\right\} \\
& +\sum_{i=1}^{n-1}\left[\prod_{k=1}^{i} U^{i}\right] \cdot \operatorname{Pr}\left\{y_{1, i+1}\right\} \cdot \operatorname{Pr}\left\{y_{2, i+1}\right\}
\end{aligned}
$$

Lemma 1.

$$
\begin{gathered}
\operatorname{Pr}\left\{x_{j, i+1}\right\} \cdot \operatorname{Pr}\left\{y_{j, i}\right\}-\operatorname{Pr}\left\{x_{j, i}\right\} \cdot \operatorname{Pr}\left\{y_{j, i+1}\right\} \geq 0 \Rightarrow \\
p_{j, i} \leq p_{j, i+1}, \text { for } j=1,2, \text { and } i=1,2, \ldots, n-1 .
\end{gathered}
$$

Proof: See appendix A.2.

Theorem 2. For CRB in conversation, the list ordered from smallest failure probability to largest failure probability, is at least as reliable as any other list of $n$ versions.

3.1 Mathematical Programming Formulation

$$
\begin{aligned}
U^{k} & \equiv\left[\sum_{i=1}^{n} \operatorname{Pr}\left\{x_{1, i}\right\} \cdot \gamma_{1: i, k}\right] \cdot\left[\sum_{i=1}^{n} \operatorname{Pr}\left\{x_{2, i}\right\} \cdot \gamma_{2: i, k}\right] \\
+ & {\left[\sum_{i=1}^{n} \operatorname{Pr}\left\{y_{1, i}\right\} \cdot \gamma_{1: i, k}\right] \cdot\left[\sum_{i=1}^{n} \operatorname{Pr}\left\{x_{2, i}\right\} \cdot \gamma_{2: i, k}\right] } \\
+ & {\left[\sum_{i=1}^{n} \operatorname{Pr}\left\{x_{1, i}\right\} \cdot \gamma_{1: i, k}\right] \cdot\left[\sum_{i=1}^{n} \operatorname{Pr}\left\{y_{2, i}\right\} \cdot \gamma_{2: i, k}\right] }
\end{aligned}
$$

The reliability of the $2 \mathrm{RB}$ in conversation is:

$$
\begin{aligned}
R_{\mathrm{c}} & =\left[\sum_{i=1}^{n} \operatorname{Pr}\left\{y_{1, i}\right\} \cdot \gamma_{1: i, 1}\right] \cdot\left[\sum_{i=1}^{n} \operatorname{Pr}\left\{y_{2, i}\right\} \cdot \gamma_{2: i, 1}\right] \\
+ & {\left[\sum_{i=1}^{n-1}\left(\prod_{k=1}^{i} U^{k}\right)\right] \cdot\left[\sum_{l=1}^{n} \operatorname{Pr}\left\{y_{1, l}\right\} \cdot \gamma_{1: l, i+1}\right] } \\
& \cdot\left[\sum_{l=1}^{n} \operatorname{Pr}\left\{y_{2, l}\right\} \cdot \gamma_{2: l, i+1}\right]
\end{aligned}
$$

The optimization problem is:

$\max \left[R_{\mathrm{c}}\right]$
Subject to:

$$
\begin{aligned}
& {\left[\sum_{j=1}^{n} \sum_{k=1}^{n} c_{1, j} \cdot \gamma_{1: j, k}\right]+\sum_{j=1}^{n} \sum_{k=1}^{n} c_{2, j} \cdot \gamma_{2: j, k} \leq B} \\
& \sum_{i=1}^{n} \gamma_{2: i, k} \leq 1, \text { for } k=1,2, \ldots, n \\
& \sum_{k=1}^{n} \gamma_{2: i, k} \leq 1, \text { for } i=1,2, \ldots, n \\
& \sum_{i=1}^{n} \gamma_{1: i, k} \leq 1, \text { for } k=1,2, \ldots, n \\
& \sum_{k=1}^{n} \gamma_{1: i, k} \leq 1, \text { for } i=1,2, \ldots, n \\
& \sum_{i=1}^{n} \sum_{k=1}^{n} \gamma_{2: i, k}=\sum_{i=1}^{n} \sum_{k=1}^{n} \gamma_{1: i, k}
\end{aligned}
$$

The objective function (19) maximizes reliability. Constraint (20) guarantees that the total expenditure does not exceed the budget. Constraints (21), (23) reflect that if a version is executed in RB-2, RB-1, respectively, that version occupies at most one position.

Constraints (22), (24) reflect that each position can be occupied by at most 1 version in $R B-2, R B-1$, respectively.

Constraint (25) guarantees that the same number of versions are selected in RB-1 \& RB-2.

\subsection{The B\&B Procedure}

This procedure can solve the optimization problem in section 3.1. Without any loss of generality we assume that, in each block, the versions are arranged from the smallest failure probability to the largest failure probability. At tree-level \#1 it is decided whether version \#1 of block \#1 is selected or not.

If version $\# 1$ is selected:

$\gamma_{1: 1,1}=1$, and $\gamma_{1: 1, k}=0$ for $k=2,3, \ldots, n$.

If version $\# 1$ is not selected:

$\gamma_{1: 1, k}=0$ for $k=1,2, \ldots, n$.

At tree-level \#2 it is determined whether to select version \#2:

if version \#1 is already selected then we select version \#2 or not; if version \#2 is selected:

$\gamma_{1: 1,1}=\gamma_{1: 2,2}=1$, and $\gamma_{1: 2, k}=0$ for $k=3,4, \ldots, n$.

Etc.

Once all the versions of block \#1 are considered it is decided whether to select version \#1 of the block \#2 in a similar way as explained in section 2.3. Continue until all versions of the block \#2 are considered.

For each partial solution, LB \& UB can be computed in a similar way to those computed in section 2.3. Any partial solution can be eliminated (fathomed) from further consideration by using the conditions explained in section 2.3 , with the addition that unfeasibility of a partial solution can occur also if there is no possibility to satisfy constraint (25). 


\subsection{Example}

Figure 2 shows the $\mathrm{B} \& \mathrm{~B}$ tree for this example. The initial LB is the objective function value of the solution that includes all the versions in the 2 blocks, except for version \#4 in RB-1 and version \#1 in RB-2, ie,

$\gamma_{1: 1,1}=\gamma_{1: 2,2}=\gamma_{1: 3,3}=\gamma_{2: 2,1}=\gamma_{2: 3,2}=\gamma_{2: 4,3}=1$

; and 0 otherwise

with objective function $=0.935189$.

Node 18 is fathomed because, for the partial solution: $\gamma_{1: 1,1}=\gamma_{1: 2,2}=\gamma_{1: 3,3}=\gamma_{1: 4,4}=1, \gamma_{2: 2,1}=0$, constraint (25) cannot hold with any solution that includes it. This is also the reason why node 24 is fathomed. The first time that 'an improved LB is found' is at node 26 which corresponds to the solution: versions $1,2,3$ of RB-1 and versions $1,3,4$ of $\mathrm{RB}-2$ are selected:

$\gamma_{1: 1,1}=\gamma_{1: 2,2}=\gamma_{1: 3,3}=\gamma_{2: 1,1}=\gamma_{2: 3,2}=\gamma_{2: 4,3}=1$, ; and 0 otherwise

with objective function $=0.952518$. This is also the optimal solution.

\section{LIMITATIONS}

The models require point estimates of all reliabilities. It is assumed that the various versions are $s$-independent. These models should be modified to model software systems with $s$-dependent versions for which reliabilities are estimated in the time domain. Our models neglect the time it takes to transfer data from one RB to another; these models should be modified to consider this time, as well as the mean time of running the versions - because they are important for systems like avionics and nuclear reactors.

\section{ACKNOWLEDGMENT}

We are pleased to thank the Associate Editor, Dr. M.A. Vouk, and the referees for their constructive comments. This work was supported by a grant from the NSERC.

\section{APPENDIX}

\section{A.1 Proof of Theorem 1}

There are 2 cases:

- Case 1 assumes that both lists are identical except for versions $l$ and $l+l$ in RB-2.

- Case 2 assumes that both lists are identical except for versions $l$ and $l+1$ in RB-1.

\section{A.1.1 Case 1}

Let $R_{\mathrm{rb}}^{k}$ be the reliability of CRB for list $k, k=1,2$.

list $1:\{(1,1), \ldots,(1, l),(1, l+1), \ldots,(1, n)$;

$(2,1), \ldots,(2, l-1),(2, l),(2, l+1), \ldots,(2, n)\}$

list 2: $\{(1,1), \ldots,(1, l),(1, l+1), \ldots,(1, n)$;

$(2,1), \ldots,(2, l-1),(2, l+1),(2, l),(2, l+2), \ldots,(2, n)\}$

$$
\begin{aligned}
& R_{\mathrm{rb}}^{1}=R_{1} \cdot \operatorname{Pr}\left\{y_{2,1}\right\} \\
& +\sum_{k=1}^{l-2} \sum_{j_{1}=2}^{n-k+1} \sum_{j_{2}=j_{1}+1}^{n-k} \cdots \sum_{j_{k}=j_{k-1}+1}^{n} R_{1}^{j_{1}^{-}} \cdot R_{1}^{j_{1}, j_{2}} \\
& +\sum_{j_{1}=2} \sum_{j_{2}=j_{1}+1}^{n-l+2} R_{1}^{j_{k-1}, j_{k}} \cdot R_{1}^{j_{k}^{+}} \cdot U_{k} \cdot \operatorname{Pr}\left\{y_{2, k+1}\right\} \\
& +\sum_{j_{1}=j_{k-1}+1}^{n-l+1} R_{1}^{j_{1}^{-}} \cdot R_{1}^{j_{1}, j_{2}} \\
& \cdots R_{1}^{j_{k-1}, j_{k}} \cdot R_{1}^{j_{k}^{+}} \cdot U_{l-1} \cdot \operatorname{Pr}\left\{y_{2, l}\right\} \\
& +\sum_{j_{2}=j_{1}+1}^{n-l+1} \sum_{j_{k}=j_{k-1}+1}^{n} R_{1}^{j_{1}^{-}} \cdot R_{1}^{j_{1}, j_{2}} \\
& \cdots R_{1}^{j_{k-1}, j_{k}} \cdot R_{1}^{j_{k}^{+}} \cdot U_{l} \cdot \operatorname{Pr}\left\{y_{2, l+1}\right\} \\
& \sum_{j_{1}=2}^{n-k+1} \sum_{j_{2}=j_{1}+1}^{n-k} \sum_{j_{k}=j_{k-1}+1}^{n} R_{1}^{j_{1}^{-}} \cdot R_{1}^{j_{1}, j_{2}}
\end{aligned}
$$

$$
\begin{aligned}
R_{\mathrm{rb}}^{2} & =R_{1} \cdot \operatorname{Pr}\left\{y_{2,1}\right\} \\
& +\sum_{k=1}^{l-2} \sum_{j_{1}=2}^{n-k+1} \sum_{j_{2}=j_{1}+1}^{n-k} \cdots \sum_{j_{k}=j_{k-1}+1}^{n} R_{1}^{j_{1}^{-}} \cdot R_{1}^{j_{1}, j_{2}} \\
& +\sum_{j_{1}=2} \sum_{j_{2}=j_{1}+1}^{n-l+2} \cdots \sum_{j_{k}=j_{k-1}+1}^{j_{k-1}, j_{k}} \cdot R_{1}^{j_{k}^{+}} \cdot U_{k} \cdot \operatorname{Pr}\left\{y_{2, k+1}\right\} \\
& +\sum_{j_{1}=2}^{n-l+1} R_{1}^{j_{1}^{-}} \cdot R_{1}^{j_{1}, j_{2}} \\
& \sum_{j_{2}=j_{1}+1}^{n-l+1} R_{1}^{j_{k-1}, j_{k}} \cdot R_{1}^{j_{k}^{+}} \cdot U_{l-1} \cdot \operatorname{Pr}\left\{y_{2, l+1}\right\} \\
& +\sum_{k=l+1}^{n-1} \sum_{j_{k}=j_{k-1}+1}^{n} R_{1}^{j_{1}^{-}} \cdot R_{1}^{j_{1}, j_{2}} \\
\sum_{j_{1}=2}^{j_{k-1}, j_{k}} \cdot R_{1}^{j_{k}^{+}} \cdot U_{l-1} \cdot \operatorname{Pr}\left\{x_{2, l+1}\right\} \cdot \operatorname{Pr}\left\{y_{2, l}\right\} & \sum_{j_{2}=j_{1}+1}^{n-k} \cdots \sum_{j_{k}=j_{k-1}+1}^{n} R_{1}^{j_{1}^{-}} \cdot R_{1}^{j_{1}, j_{2}} \\
& \equiv \prod_{j=1}^{k} \operatorname{Pr}\left\{R_{1}^{j_{k-1}, j_{k}} \cdot R_{1}^{j_{k}^{+}} \cdot U_{k} \cdot \operatorname{Pr}\left\{y_{2, k+1}\right\}\right.
\end{aligned}
$$

Therefore,

$$
\begin{aligned}
& R_{\mathrm{rb}}^{1} \geq R_{\mathrm{rb}}^{2} \Leftrightarrow \bar{t}_{2,2} \cdot\left[p_{2, l+1}\right. \\
& \left.\quad-p_{2, l}\right]-\bar{t}_{2,1} \cdot \bar{t}_{2,2} \cdot \bar{t}_{2,3} \cdot\left[p_{2, l+1}-p_{2, l}\right] \geq 0
\end{aligned}
$$

Inequality $(\mathrm{A}-3)$ is true iff $p_{2, l+1} \geq p_{2, l}$.

\section{A.1.2 Case 2}

Consider lists 3 \& 4 :

list $3:\{(1,1), \ldots,(1, l-1),(1, l),(1, l+1), \ldots,(1, n)$;

$$
(2,1), \ldots,(2, l),(2, l+1), \ldots,(2, n)\}
$$




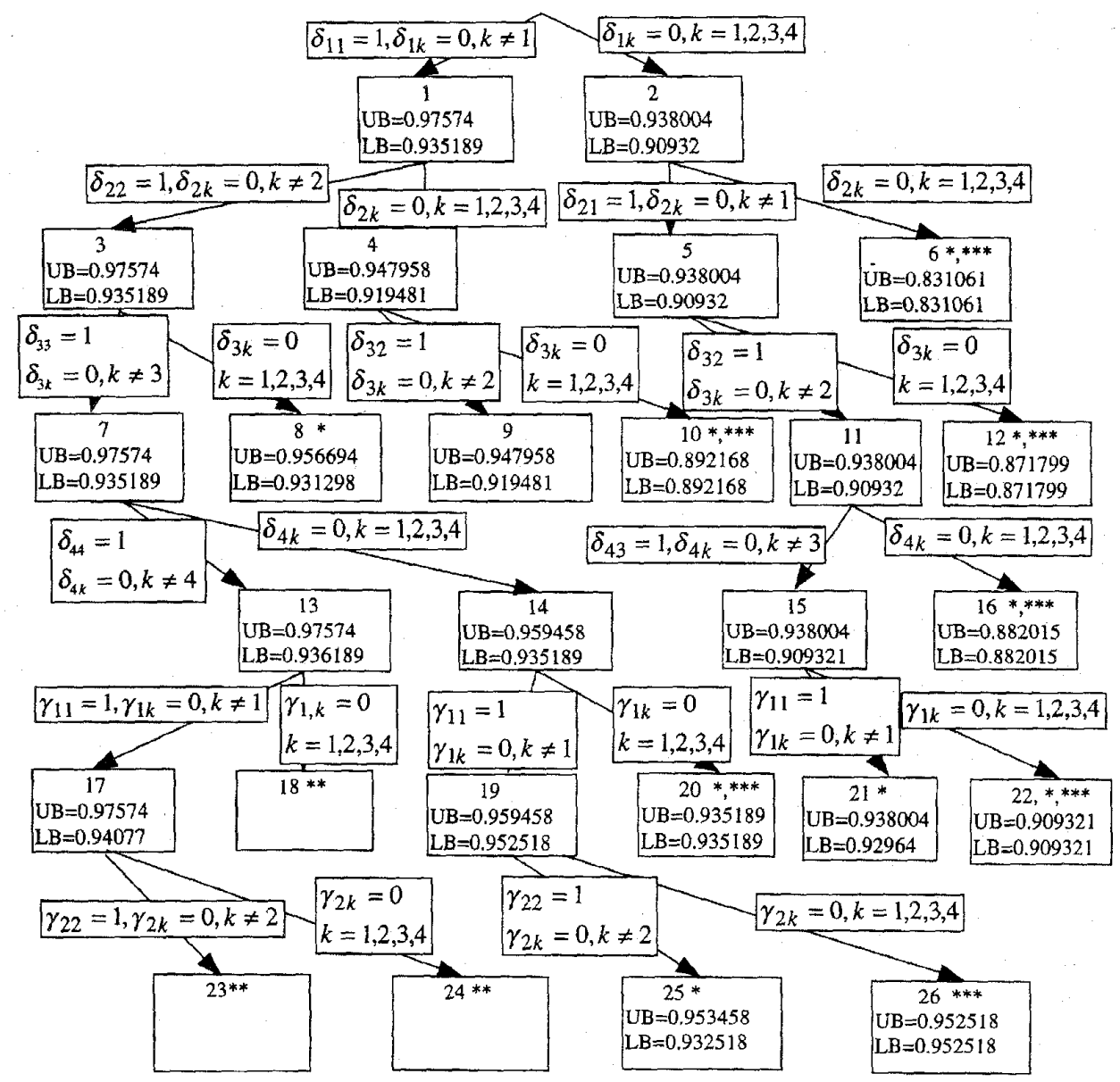

Figure 2: The B\&B for Example 3.3 Which Has CRB In Conversation

$$
\left[\gamma_{j k} \equiv \gamma_{2: j, k}, \quad \delta_{j k} \equiv \gamma_{1: j, k}\right]
$$

list 4: $\{(1,1), \ldots,(1, l-1),(1, l+1),(1, l),(1, l+2)$,

$$
\ldots,(1, n) ;(2,1), \ldots,(2, l),(2, l+1), \ldots,(2, n)\}
$$

Let $R_{\mathrm{rb}}^{k}(n)$ be the reliability of CRB for list $k=3,4$. We prove, using induction, that:

$$
R_{\mathrm{rb}}^{3}(n) \geq R_{\mathrm{rb}}^{4}(n) \text { iff } p_{1, l+1} \geq p_{1, l} .
$$

Let $n=2$.

Consider the list of versions $\{(1,1),(1,2) ;(2,1),(2,2)\}$. Then,

$$
\begin{aligned}
& R_{\mathrm{rb}}^{3}(2)=\operatorname{Pr}\left\{y_{1,1}\right\} \cdot \operatorname{Pr}\left\{y_{2,1}\right\} \\
& \quad+\operatorname{Pr}\left\{x_{1,1}\right\} \cdot \operatorname{Pr}\left\{y_{1,2}\right\} \cdot \operatorname{Pr}\left\{y_{2,1}\right\} \\
& \quad+\operatorname{Pr}\left\{y_{1,1}\right\} \cdot \operatorname{Pr}\left\{x_{2,1}\right\} \cdot \operatorname{Pr}\left\{y_{1,2}\right\} \cdot \operatorname{Pr}\left\{y_{2,2}\right\}
\end{aligned}
$$

Consider the list of versions $\{(1,2),(1,1) ;(2,1),(2,2)\}$.
Then,

$$
\begin{aligned}
& R_{\mathrm{rb}}^{4}(2)=\operatorname{Pr}\left\{y_{1,2}\right\} \cdot \operatorname{Pr}\left\{y_{2,1}\right\} \\
& \quad+\operatorname{Pr}\left\{x_{1,2}\right\} \cdot \operatorname{Pr}\left\{y_{1,1}\right\} \cdot \operatorname{Pr}\left\{y_{2,1}\right\} \\
& \quad+\operatorname{Pr}\left\{y_{1,2}\right\} \cdot \operatorname{Pr}\left\{x_{2,1}\right\} \cdot \operatorname{Pr}\left\{y_{1,1}\right\} \cdot \operatorname{Pr}\left\{y_{2,2}\right\} \\
& \quad R_{\mathrm{rb}}^{3}(2) \geq R_{\mathrm{rb}}^{4}(2) \Leftrightarrow R_{\mathrm{rb}}^{3}(2)-R_{\mathrm{rb}}^{4}(2) \geq 0
\end{aligned}
$$

or iff

$$
\begin{aligned}
& \operatorname{Pr}\left\{y_{2,1}\right\} \cdot\left[\operatorname{Pr}\left\{y_{1,1}\right\}-\operatorname{Pr}\left\{y_{1,2}\right\}\right]+\operatorname{Pr}\left\{y_{2,1}\right\} \\
& \quad \cdot\left[\operatorname{Pr}\left\{x_{1,1}\right\} \cdot \operatorname{Pr}\left\{y_{1,2}\right\}-\operatorname{Pr}\left\{x_{1,2}\right\} \cdot \operatorname{Pr}\left\{y_{1,1}\right\}\right] \geq 0
\end{aligned}
$$

This inequality is true iff $p_{1,2} \geq p_{1,1}$. Thus,

$$
R_{\mathrm{rb}}^{3}(2) \geq R_{\mathrm{rb}}^{4}(2) \text {, iff } p_{1,2} \geq p_{1,1} \text {. }
$$

Suppose the theorem holds for $n$; we prove it for $n+1$. Let $R^{3}(n+1), R^{4}(n+1)$ be respectively the sum of all 
possible terms of $R_{\mathrm{rb}}^{3}(n+1), R_{\mathrm{rb}}^{4}(n+1)$ that correspond to versions $(1, n+1),(2, n+1)$. For example, for $n+1=3$,

$$
\begin{aligned}
& R^{3}(3)=\operatorname{Pr}\left\{x_{1,1}\right\} \cdot \operatorname{Pr}\left\{x_{1,2}\right\} \cdot \operatorname{Pr}\left\{y_{1,3}\right\} \cdot \operatorname{Pr}\left\{y_{2,1}\right\} \\
& +\operatorname{Pr}\left\{y_{1,1}\right\} \cdot \operatorname{Pr}\left\{x_{2,1}\right\} \cdot \operatorname{Pr}\left\{x_{1,2}\right\} \cdot \operatorname{Pr}\left\{y_{1,3}\right\} \cdot \operatorname{Pr}\left\{y_{2,2}\right\} \\
& +\operatorname{Pr}\left\{x_{1,1}\right\} \cdot \operatorname{Pr}\left\{y_{1,2}\right\} \cdot \operatorname{Pr}\left\{x_{2,1}\right\} \cdot \operatorname{Pr}\left\{y_{1,3}\right\} \cdot \operatorname{Pr}\left\{y_{2,2}\right\} \\
& +\operatorname{Pr}\left\{y_{1,1}\right\} \cdot \operatorname{Pr}\left\{x_{2,1}\right\} \cdot \operatorname{Pr}\left\{y_{1,2}\right\} \cdot \operatorname{Pr}\left\{x_{2,2}\right\} \cdot \operatorname{Pr}\left\{y_{1,3}\right\} \\
& \cdot \operatorname{Pr}\left\{y_{2,3}\right\} \\
& R^{4}(3)=\operatorname{Pr}\left\{x_{1,2}\right\} \cdot \operatorname{Pr}\left\{x_{1,1}\right\} \cdot \operatorname{Pr}\left\{y_{1,3}\right\} \cdot \operatorname{Pr}\left\{y_{2,1}\right\} \\
& +\operatorname{Pr}\left\{y_{1,2}\right\} \cdot \operatorname{Pr}\left\{x_{2,1}\right\} \cdot \operatorname{Pr}\left\{x_{1,1}\right\} \cdot \operatorname{Pr}\left\{y_{1,3}\right\} \cdot \operatorname{Pr}\left\{y_{2,2}\right\} \\
& +\operatorname{Pr}\left\{x_{1,2}\right\} \cdot \operatorname{Pr}\left\{y_{1,1}\right\} \cdot \operatorname{Pr}\left\{x_{2,1}\right\} \cdot \operatorname{Pr}\left\{y_{1,3}\right\} \cdot \operatorname{Pr}\left\{y_{2,2}\right\} \\
& +\operatorname{Pr}\left\{y_{1,2}\right\} \cdot \operatorname{Pr}\left\{x_{2,1}\right\} \cdot \operatorname{Pr}\left\{y_{1,1}\right\} \cdot \operatorname{Pr}\left\{x_{2,2}\right\} \cdot \operatorname{Pr}\left\{y_{1,3}\right\} \\
& \cdot \operatorname{Pr}\left\{y_{2,3}\right\} \cdot
\end{aligned}
$$

Note that $R^{3}(3)=R^{4}(3)$; this is true for any $n$, ie, $R^{3}(n)=R^{4}(n)$. Obviously,

$$
\begin{aligned}
& R_{\mathrm{rb}}^{3}(n+1)=R_{\mathrm{rb}}^{3}(n)+R^{3}(n+1) \\
& R_{\mathrm{rb}}^{4}(n+1)=R_{\mathrm{rb}}^{4}(n)+R^{4}(n+1)
\end{aligned}
$$

By induction,

$R_{\mathrm{rb}}^{3}(n) \geq R_{\mathrm{rb}}^{4}(n)$ iff $p_{1, l} \leq p_{1, l+1}$.

Q.E.D.

\section{A.2 Proof of Lemma 1}

The proof is obvious, since the 1.h.s. of the expression can be written as:

$$
\bar{t}_{j, 1} \cdot \bar{t}_{j, 2} \cdot \bar{t}_{j, 3} \cdot\left[p_{j, i+1}-p_{j, i}\right]
$$

$$
\text { Q.E.D. }
$$

\section{A.3 Proof of Theorem 2}

There are 2 cases:

- Case 1 assumes that both lists are identical except for versions $i$ and $i+1$ in RB- 1 .

- Case 2 assumes that both lists are identical except for versions $i$ and $i+1$ in RB-2.

\section{A.3.1 Case 1}

Let $R_{\mathrm{c}}^{k}$ be the reliability of CRB in conversation for list $k=1,2$.

list $1:\{(1,1), \ldots,(1, i-1),(1, i),(1, l+1), \ldots,(1, n)$;

$$
(2,1), \ldots,(2, i),(2, i+1), \ldots,(2, n)\}
$$

list $2:\{(1,1), \ldots,(1, i-1),(1, i+1),(1, i)$,

$$
(1, i+2), \ldots,(1, n) ;(2,1), \ldots,(2, i),(2, i+1), \ldots,(2, n)\}
$$

$$
\begin{aligned}
R_{\mathrm{c}}^{1} & =\operatorname{Pr}\left\{y_{1,1}\right\} \cdot \operatorname{Pr}\left\{y_{2,1}\right\} \\
& +\sum_{j=1}^{i-2}\left[\prod_{k=1}^{j} U^{k}\right] \cdot \operatorname{Pr}\left\{y_{1, j+1}\right\} \cdot \operatorname{Pr}\left\{y_{2, j+1}\right\} \\
& +\left[\prod_{k=1}^{i-1} U^{k}\right] \cdot \operatorname{Pr}\left\{y_{1, i}\right\} \cdot \operatorname{Pr}\left\{y_{2, i}\right\} \\
& +\left[\prod_{k=1}^{i-1} U^{k}\right] \cdot U^{i} \cdot \operatorname{Pr}\left\{y_{1, i+1}\right\} \cdot \operatorname{Pr}\left\{y_{2, i+1}\right\} \\
& +\sum_{j=i+1}^{n-1}\left[\prod_{k=1}^{j} U^{k}\right] \cdot \operatorname{Pr}\left\{y_{1, j+1}\right\} \cdot \operatorname{Pr}\left\{y_{2, j+1}\right\}
\end{aligned}
$$

$$
\begin{aligned}
R_{\mathrm{c}}^{2} & =\operatorname{Pr}\left\{y_{1,1}\right\} \cdot \operatorname{Pr}\left\{y_{2,1}\right\} \\
& +\sum_{j=1}^{i-2}\left[\prod_{k=1}^{j} U^{k}\right] \cdot \operatorname{Pr}\left\{y_{1, j+1}\right\} \cdot \operatorname{Pr}\left\{y_{2, j+1}\right\} \\
& +\left[\prod_{k=1}^{i-1} U^{k}\right] \cdot \operatorname{Pr}\left\{y_{1, i+1}\right\} \cdot \operatorname{Pr}\left\{y_{2, i}\right\} \\
& +\left[\prod_{k=1}^{i-1} U^{k}\right] \cdot U\left(i_{1}\right) \cdot \operatorname{Pr}\left\{y_{1, i}\right\} \cdot \operatorname{Pr}\left\{y_{2, i+1}\right\} \\
& +U\left(i_{1}\right) \cdot U\left(i_{2}\right) \\
& \cdot \sum_{j=i+1}^{n-1}\left[\prod_{k=1}^{j} U_{k \neq i, i+1}^{k}\right] \cdot \operatorname{Pr}\left\{y_{1, j+1}\right\} \cdot \operatorname{Pr}\left\{y_{2, j+1}\right\}
\end{aligned}
$$

$$
\begin{aligned}
& U\left(i_{1}\right) \equiv \operatorname{Pr}\left\{x_{1, i+1}\right\} \cdot \operatorname{Pr}\left\{x_{2, i}\right\}+\operatorname{Pr}\left\{y_{1, i+1}\right\} \cdot \operatorname{Pr}\left\{x_{2, i}\right\} \\
& \quad+\operatorname{Pr}\left\{x_{1, i+1}\right\} \cdot \operatorname{Pr}\left\{y_{2, i}\right\} \\
& U\left(i_{2}\right) \equiv \operatorname{Pr}\left\{x_{1, i}\right\} \cdot \operatorname{Pr}\left\{x_{2, i+1}\right\}+\operatorname{Pr}\left\{y_{1, i}\right\} \cdot \operatorname{Pr}\left\{x_{2, i+1}\right\} \\
& \quad+\operatorname{Pr}\left\{x_{1, i}\right\} \cdot \operatorname{Pr}\left\{y_{2, i+1}\right\}
\end{aligned}
$$

Let:

$C_{1} \equiv \prod_{k=1}^{i-1} U^{k}$

$C \equiv\left[\operatorname{Pr}\left\{y_{1, i}\right\} \cdot \operatorname{Pr}\left\{x_{1, i+1}\right\}-\operatorname{Pr}\left\{y_{1, i+1}\right\} \cdot \operatorname{Pr}\left\{x_{1, i}\right\}\right]$

- $\left[\operatorname{Pr}\left\{y_{2, i}\right\} \cdot \operatorname{Pr}\left\{x_{2, i+1}\right\}-\operatorname{Pr}\left\{y_{2, i+1}\right\} \cdot \operatorname{Pr}\left\{x_{2, i}\right\}\right] \cdot C_{1} \cdot A$;

$$
A \equiv \sum_{j=i+1}^{n-1}\left[\prod_{k=i+2}^{j} U^{k}\right] \cdot \operatorname{Pr}\left\{y_{1, j+1}\right\} \cdot \operatorname{Pr}\left\{y_{2, j+1}\right\}
$$

Using lemma 1 and since $A \leq 1$ :

$C \leq C_{1} \cdot\left[\operatorname{Pr}\left\{y_{1, i}\right\} \cdot \operatorname{Pr}\left\{x_{1, i+1}\right\}-\operatorname{Pr}\left\{y_{1, i+1}\right\} \cdot \operatorname{Pr}\left\{x_{1, i}\right\}\right]$

$\cdot\left[\operatorname{Pr}\left\{y_{2, i}\right\} \cdot \operatorname{Pr}\left\{x_{2, i+1}\right\}-\operatorname{Pr}\left\{y_{2, i+1}\right\} \cdot \operatorname{Pr}\left\{x_{2, i}\right\}\right]$

Let:

$I \equiv R_{\mathrm{c}}^{1}-R_{\mathrm{c}}^{2}$.

From $(\mathrm{A}-5)-(\mathrm{A}-7)$ :

$$
\begin{aligned}
I= & C_{1} \cdot \operatorname{Pr}\left\{y_{2, i}\right\} \cdot\left[\operatorname{Pr}\left\{y_{1, i}\right\}-\operatorname{Pr}\left\{y_{1, i+1}\right\}\right] \\
& -C_{1} \cdot \operatorname{Pr}\left\{y_{2, i+1}\right\} \cdot\left[\operatorname{Pr}\left\{x_{1, i+1}\right\} \cdot \operatorname{Pr}\left\{y_{1, i}\right\}\right. \\
& \left.-\operatorname{Pr}\left\{x_{1, i}\right\} \cdot \operatorname{Pr}\left\{y_{1, i+1}\right\}\right] \cdot\left[\operatorname{Pr}\left\{x_{2, i}\right\}+\operatorname{Pr}\left\{y_{2, i}\right\}\right]-C
\end{aligned}
$$


Thus,

$$
\begin{aligned}
& I \geq 0 \Leftrightarrow \\
& \operatorname{Pr}\left\{y_{2, i}\right\} \cdot\left[\operatorname{Pr}\left\{y_{1, i}\right\}-\operatorname{Pr}\left\{y_{1, i+1}\right\}\right] \\
& \quad-\operatorname{Pr}\left\{y_{2, i+1}\right\} \cdot\left[\operatorname{Pr}\left\{x_{2, i}\right\}+\operatorname{Pr}\left\{y_{2, i}\right\}\right] \\
& \quad \cdot\left[\operatorname{Pr}\left\{x_{1, i+1}\right\} \cdot \operatorname{Pr}\left\{y_{1, i}\right\}-\operatorname{Pr}\left\{x_{1, i}\right\} \cdot \operatorname{Pr}\left\{y_{1, i+1}\right\}\right] \\
& \quad-\left[\operatorname{Pr}\left\{x_{1, i+1}\right\} \cdot \operatorname{Pr}\left\{y_{1, i}\right\}-\operatorname{Pr}\left\{x_{1, i}\right\} \cdot \operatorname{Pr}\left\{y_{1, i+1}\right\}\right] \\
& \quad \cdot\left[\operatorname{Pr}\left\{x_{2, i+1}\right\} \cdot \operatorname{Pr}\left\{y_{2, i}\right\}-\operatorname{Pr}\left\{x_{2, i}\right\} \cdot \operatorname{Pr}\left\{y_{2, i+1}\right\}\right] \\
&=\operatorname{Pr}\left\{y_{2, i}\right\} \cdot\left[\operatorname{Pr}\left\{y_{1, i}\right\}-\operatorname{Pr}\left\{y_{1, i+1}\right\}\right] \\
& \quad-\operatorname{Pr}\left\{y_{2, i}\right\} \cdot\left[\operatorname{Pr}\left\{x_{2, i+1}\right\}+\operatorname{Pr}\left\{y_{2, i+1}\right\}\right] \\
& \quad \cdot\left[\operatorname{Pr}\left\{x_{1, i+1}\right\} \cdot \operatorname{Pr}\left\{y_{1, i}\right\}-\operatorname{Pr}\left\{x_{1, i}\right\} \cdot \operatorname{Pr}\left\{y_{1, i+1}\right\}\right] \geq 0
\end{aligned}
$$

But because,

$$
\begin{aligned}
& \operatorname{Pr}\left\{x_{2, i+1}\right\}+\operatorname{Pr}\left\{y_{2, i+1}\right\} \leq 1, \text { then } I \geq 0, \text { iff } \\
& \operatorname{Pr}\left\{y_{1, i}\right\}-\operatorname{Pr}\left\{y_{1, i+1}\right\}+\operatorname{Pr}\left\{y_{1, i+1}\right\} \cdot \operatorname{Pr}\left\{x_{1, i}\right\} \\
& \quad-\operatorname{Pr}\left\{y_{1, i}\right\} \cdot \operatorname{Pr}\left\{x_{1, i+1}\right\} \geq 0 .
\end{aligned}
$$

Using (A-4),

$$
\begin{aligned}
& \operatorname{Pr}\left\{y_{1, i}\right\}-\operatorname{Pr}\left\{y_{1, i+1}\right\}+\operatorname{Pr}\left\{y_{1, i+1}\right\} \cdot \operatorname{Pr}\left\{x_{1, i}\right\} \\
& \quad-\operatorname{Pr}\left\{y_{1, i}\right\} \cdot \operatorname{Pr}\left\{x_{1, i+1}\right\} \\
& =\bar{t}_{1,2} \cdot\left[p_{1, i+1}-p_{1, i}\right]-\bar{t}_{1,1} \cdot \bar{t}_{1,2} \cdot \bar{t}_{1,3} \cdot\left[p_{1, i+1}-p_{1, i}\right] \geq 0 \\
& \Leftrightarrow p_{1, i} \leq p_{1, i+1} .
\end{aligned}
$$

\section{A.3.2 Case 2}

Let $R_{\mathrm{c}}^{k}$ be the reliability of CRB in conversation for list $k, k=3,4$.

list . $3:\{(1,1), \ldots,(1, i),(1, i+1), \ldots,(1, n)$;

$(2,1), \ldots,(2, i-1),(2, i),(2, i+1), \ldots,(2, n)\}$

list $4:\{(1,1), \ldots,(1, i),(1, i+1), \ldots,(1, n)$;

$(2,1), \ldots,(2, i-1),(2, i+1),(2, i),(2, i+2), \ldots,(2, n)\}$

The proof is similar to case 1 .

Q.E.D.

\section{REFERENCES}

[1] O. Berman, U. Dinesh Kumar, "Optimization models for recovery block schemes", To appear in European J. Operational Research.

[2] F. Belli, P. Jedrzejowicz, "Reliability modelling of the fault tolerant programs", Proc. Fifth IASTED Int'l Conf. - Reliability and Quality Control, 1989, pp 53 - 56. Lugano.

[3] F. Belli, P. Jedrzejowicz, "An approach to reliability optimization of software with redundancy", IEEE Trans. Software Eng'g, vol 19, num 11, 1993, pp 1119 - 1123.

[4] J.B. Dugan, "Software system analysis using fault trees", [8: pp 615-659].

[5] H. Hecht, "Fault tolerant software", IEEE Trans. Reliability, vol 28, 1979 Aug, pp $227-232$.

[6] P. Jalote, Fault Tolerance in Distributed Systems, 1994; Prentice Hall.

[7] K.H. Kim, "The distributed recovery block scheme", Software Fault Tolerance, 1995, pp $189-209$; John Wiley \& Sons.
[8] Handbook of Software Reliability Engineering (M.R. Lyu, $E d)$, 1996; IEEE Computer Society Press.

[9] Software Fault Tolerance (M.R. Lyu, Ed), 1995; John Wiley \& Sons.

[10] D.F. McAllister, M.A. Vouk, "Fault-tolerant software reliability engineering", [8: pp 567 - 614].

[11] V.F. Nicola, "Checkpointing and the modeling of program execution time", [9: pp 167-188]

[12] B. Randell, "System structure for software fault tolerance", IEEE Trans. Software Eng'g, vol 13, num 5, 1975, pp $582-592$.

[13] D.L. Russel, "State restoration in systems of communicating processes", IEEE Trans. Software Eng'g, vol 6, num 2, 1980, pp $183-194$.

[14] R.E. Scott, J.W. Gault, D.F. McAllister, "Fault-tolerant software reliability modelling", IEEE Trans. Software Eng'g, vol 13, num 5, 1987, pp 582- 595 .

\section{AUTHORS}

Prof. Oded Berman; Joseph L. Rotman School of Management; Univ. of Toronto; 105 St. George St; Toronto, Ontario M5S 3E6 CANADA.

Internet (e-mail): berman@mgmt.utoronto.ca

Oded Berman is a full professor and the former Associate Dean of Programs at the Joseph L. Rotman School of Management at the University of Toronto. He received his $\mathrm{PhD}$ (1978) in Operations Research from the Massachusetts Institute of Technology. He had been with the Electronic Systems Lab at MIT, the Univ. of Calgary, and the Univ. of Massachusetts at Boston, where he was also the Chairman of the Dept. of Management Sciences. He has published over 90 articles and has contributed to several books in his field. His main research interests include operations management in the service industry, location theory, network models, and software reliability. He is the Area Editor of services and military for Operations Research, an Associate Editor of Management Science and Transportation Science, and a member of the editorial boards for Computers and Operations Research and the Journal of Service Research.

Dr. U. Dinesh Kumar; Centre M.I.R.C.E; University of Exeter; Exeter EX4 4QF UNITED KINGDOM.

Internet (e-mail): d.k.unnikrishnan@ex.ac.uk

Dinesh Kumar is a Lecturer in Reliability Engineering at the Centre for Management of Industrial Reliability, Cost and Effectiveness at the University of Exeter, UK. He holds a Doctors Degree in Mathematics from the Indian Institute of Technology, Bombay, and Masters Degree in Operations Research from Bharathiyar University, India. He has over 50 research publications in reliability, software reliability, maintenance, and logistics. Dr. Kumar is a member of SOLE (The International Society of Logistics Engineers) and Indian Statistical Association. His research sponsors include EPSRC, CSIR, and Rolls Royce Military Aero Engines Ltd.

Manuscript TR96-164 received: 1996 December 3; revised: 1998 July 7

Responsible editor: M.A. Vouk

Publisher Item Identifier S 0018-9529(98)09657-2 